\title{
Atomic effect algebras with compression bases
}

\author{
Dan Caragheorgheopol ${ }^{1}$, Josef Tkadlec $^{2}$ \\ ${ }^{1}$ Department of Mathematics and Informatics, Technical University of Civil Engineering in \\ Bucharest, 124 Lacul Tei blv., RO-020396, Romania and "Ilie Murgulescu" Institute of Phys- \\ ical Chemistry, Romanian Academy, 202 Splaiul Independentei, RO-060021 Bucharest, Ro- \\ mania; e-mail:dancaraghe@gmail.com \\ ${ }^{2}$ Department of Mathematics, Faculty of Electrical Engineering, Czech Technical University \\ in Prague, 166 27 Prague, Czech Republic; e-mail: tkadlec@fel.cvut.cz
}

(Received 13 August 2010; accepted 14 December 2010; published online 20 January 2011)

(C) 2011 American Institute of Physics. [doi:10.1063/1.3533918]

Compression base effect algebras were recently introduced by Gudder [6]. They generalize sequential effect algebras [7] and compressible effect algebras [5]. The present paper focuses on atomic compression base effect algebras and the consequences of atoms being foci (so-called projections) of the compressions in the compression base. Part of our work generalizes results obtained in atomic sequential effect algebras by Tkadlec [11]. The notion of projection-atomicity is introduced and studied and several conditions that force a compression base effect algebra or the set of its projections to be Boolean are found. Finally, we apply some of these results to sequential effect algebras and strengthen a previously established result concerning a sufficient condition for them to be Boolean.

\section{Introduction}

The current framework for discussing the logical foundations of quantum mechanics is the algebraic structure of an effect algebra, which allows the study of measurements or observables that may be unsharp (see, e.g., [2]). Gudder and Greechie [7] discussed the notion of a sequential effect algebra (SEA) - an effect algebra on which a "sequential product" is defined. This sequential product satisfies a set of physically motivated axioms as it formalizes the case of sequentially performed measurements. The authors prove that the existence of a sequential product in an effect algebra is a restrictive condition, far from being met by all effect algebras.

Gudder [5] introduced the notion of a compression on an effect algebra and also of a compressible effect algebra. Although the important examples of effect algebras proves to be compressible, examples are also provided of noncompressible effect algebras.

As it turns out, the two notions (sequential effect algebra and compressible effect algebra) are somehow related, since the sequential product with a sharp element (of a SEA) defines a compression. Although the restrictions imposed by the existence of a sequential product seem stronger than those determined by compressibility, neither of the two notions is a generalization of the other, as an example of a noncompressible SEA shows [5]. However, in a later paper Gudder [6] introduced a common generalization of both SEA and compressible effect algebras, namely effect algebras having a compression base.

Tkadlec [11] proved various conditions for an atomic SEA or its set of sharp elements to be a Boolean algebra. In this paper we generalize some of these conditions to the case of effect algebras having a compression base, and also present some new ones for this more general framework. The role of the set of sharp elements of the SEA will be played by the orthomodular poset of foci (or projections) of the effect algebra's compression base.

In Sec. II, we recall some of the basic facts about effect algebras and their atoms. Section III is devoted to an introduction to compressions and their basic properties, as well as compression bases. As a particular case of compression base effect algebras, we briefly present sequential effect algebras. Sections IV and V contain results concerning mainly atomic compression base 
effect algebras. Sec. IV we establish some properties of atoms in effect algebras endowed with a compression base, mainly regarding coexistence and centrality. Then, in Sec. V, we introduce the notion of projection-atomicity which aims to be an analogue, in the framework of effect algebras with a compression base, for the property of an effect algebra of having sharp atomsused in sequential effect algebras. Consequences of projection-atomicity are studied, some of which generalize results obtained in [11. A few conditions for an atomic compression base effect algebra to be a Boolean algebra are established. Finally, we apply these results to the particular case of sequential effect algebras and find a sufficient condition for them to be Boolean algebras that strengthens previous results by Gudder and Greechie [7] and Tkadlec [11.

\section{Basics about effect algebras}

Definition 2.1 An effect algebra is an algebraic structure $(E, \oplus, \mathbf{0}, \mathbf{1})$ such that $E$ is a set, $\mathbf{0}, \mathbf{1} \in E, \oplus$ is a partial binary operation on $E$ such that for $a, b, c$ elements of $E$ the following conditions hold:

(EA1) $a \oplus b=b \oplus a$ if $b \oplus a$ is defined;

(EA2) $(a \oplus b) \oplus c=a \oplus(b \oplus c)$ if $a \oplus(b \oplus c)$ is defined;

(EA3) for every $a \in E$, there is a unique $a^{\prime} \in E$ such that $a \oplus a^{\prime}=\mathbf{1}$ (orthosupplement);

(EA4) $a=\mathbf{0}$ whenever $a \oplus \mathbf{1}$ is defined (zero-unit law).

We usually write $E$ rather than $(E, \oplus, \mathbf{0}, \mathbf{1})$, for simplicity. A partial ordering is defined on an effect algebra $E$ by $a \leq b$ if there is an element $c \in E$ such that $a \oplus c=b$. If the element $c$ exists, it is uniquely determined by $c=\left(a \oplus b^{\prime}\right)^{\prime}$ and it is denoted by $b \ominus a$. For $a, b \in E$ with $a \leq b$ we denote $[a, b]=\{c \in E: a \leq c \leq b\}$. An orthogonality relation is defined by $a \perp b$ if $a \oplus b$ exists (i.e., $\left.a \leq b^{\prime}\right)$. It is easy to check that $\mathbf{0}$ and $\mathbf{1}$ are the least and the greatest elements of $E$, respectively, that $a^{\prime \prime}=a$, and that $a \leq b$ implies $b^{\prime} \leq a^{\prime}$. Also, $a \oplus \mathbf{0}=a$ for every $a \in E$ and a cancellation law holds: $a \oplus b \leq a \oplus c$ implies $b \leq c$ for every $a, b, c \in E$. (See, e.g., Foulis and Bennett [2, Dvurečenskij and Pulmannová [1]).

Let us consider the effect algebras $E$ and $E^{\prime}$ and the mapping $J: E \rightarrow E^{\prime}$. We denote $\operatorname{Ker}(J)=\{a \in E: J(a)=\mathbf{0}\}$. We call $J$ additive if $a \perp b$ implies $J(a) \perp J(b)$ and $J(a \oplus b)=$ $J(a) \oplus J(b)$. A subset $F$ of the effect algebra $E$ is a sub-effect algebra (denoted sub-EA in the following) if $\mathbf{0}, \mathbf{1} \in F$ and $F$ is closed under operations $\oplus$ and '.

Definition 2.2 An element $a$ of an effect algebra $(E, \oplus, \mathbf{0}, \mathbf{1})$ is called:

- $\operatorname{sharp}\left(a \in E_{\mathrm{s}}\right)$ if $a \wedge a^{\prime}=\mathbf{0}$;

- principal if $b \oplus c \leq a$ whenever $b, c \leq a$ and $b \perp c$;

- central if $a$ and $a^{\prime}$ are principal and for every $b \in E$ there are $b_{1}, b_{2} \in E$ such that $b=b_{1} \oplus b_{2}$ with $b_{1} \leq a$ and $b_{2} \leq a^{\prime}$.

It is well known that central elements are principal and principal elements are sharp. The reverse implications need not hold.

Definition 2.3 An orthoalgebra is an effect algebra in which every element is sharp. An orthomodular poset $(O M P)$ is an effect algebra in which every element is principal.

Definition 2.4 Let $E$ be an effect algebra and let us denote by $n a$ the sum of $n$ copies of an element $a \in E$, if it exists. We call $E$ Archimedean if $\sup \{n \in \mathbb{N}: n a$ is defined $\}<\infty$ for every nonzero element $a \in E$.

Let us remark that every orthoalgebra is Archimedean since no nonzero element is orthogonal to itself. 
Definition 2.5 Let $E$ be an effect algebra. A system $\left(a_{i}\right)_{i \in I}$ of elements of $E$ is orthogonal if $\bigoplus_{i \in F} a_{i}$ is defined for every finite set $F \subset I$. A majorant of an orthogonal system $\left(a_{i}\right)_{i \in I}$ is an upper bound of $\left\{\bigoplus_{i \in F} a_{i}: F \subset I\right.$ is finite $\}$. The sum of an orthogonal system is its least majorant (if it exists).

An effect algebra $E$ is orthocomplete if every orthogonal system in $E$ has a sum. An effect algebra $E$ is weakly orthocomplete if every orthogonal system in $E$ has a sum or no minimal majorant.

Definition 2.6 Let $E$ be an effect algebra. Elements $a, b \in E$ coexist (denoted by $a \leftrightarrow b$ ) if there are $a_{1}, b_{1}, c \in E$ such that $a_{1} \oplus b_{1} \oplus c$ exists and $a=a_{1} \oplus c, b=b_{1} \oplus c$.

Definition 2.7 Let $E$ be an effect algebra. A minimal non-zero element of $\mathrm{E}$ is called an atom. $E$ is atomic if every non-zero element dominates an atom. $E$ is atomistic if every non-zero element is the supremum of the atoms it dominates. $E$ is determined by atoms if, for different $a, b \in E$, the sets of atoms dominated by $a$ and $b$ are different.

The relations between these notions are outlined in the following result.

Lemma 2.8 [11, Lemma 2.2] Every atomistic effect algebra is determined by atoms. Every effect algebra determined by atoms is atomic.

The converse implications do not hold [4, 11].

Proposition 2.9 [11, Corollary 2.6] Every atomic effect algebra in which each atom is sharp is an orthoalgebra.

\section{Compression bases in effect algebras}

In this section we will present a few basic facts about compression bases in effect algebras. For a detailed discussion, examples and more on their properties, the reader is refered to [6, 9 .

Definition 3.1 Let $E$ be an effect algebra. An additive map $J: E \rightarrow E$ is a retraction if $a \leq J(\mathbf{1})$ implies $J(a)=a, J(\mathbf{1})$ is then called the focus of $J$. A retraction is a compression if $J(a)=\mathbf{0}$ implies $a \leq J(\mathbf{1})^{\prime}$. Retractions $J, I$ on $E$ are supplementary if $\operatorname{Ker}(J)=I(E)$ and $\operatorname{Ker}(I)=J(E), I$ is then called a supplement of $J$. An element $p$ of $E$ is called a projection if it is the focus of some retraction on $E$.

Let us remark that a retraction $J$ is additive, hence it is order preserving. Therefore $J(a)=a$ implies $a \leq J(\mathbf{1})$. It is also easy to see that retractions are idempotent, which suggests they are generalizations of projection mappings (except that the latter are not additive).

Definition 3.2 Let $E$ be an effect algebra. A sub-EA $F$ of $E$ is normal if, for every $a, b, c \in E$ such that $a \oplus b \oplus c$ exists in $E$ and $a \oplus b, b \oplus c \in F$, it follows that $b \in F$.

Definition 3.3 Let $E$ be an effect algebra. A system $\left(J_{p}\right)_{p \in P}$ of compressions on $E$ indexed by a normal sub-EA $P$ of $E$ is called a compression base for $E$ if the following conditions hold:

(1) Each compression $J_{p}$ has the focus $p$.

(2) If $p, q, r \in P$ and $p \oplus q \oplus r$ is defined in $E$, then $J_{p \oplus r} \circ J_{r \oplus q}=J_{r}$.

Let us remark here the obvious fact that every effect algebra has a trivial compression base $\left\{J_{\mathbf{0}}, J_{\mathbf{1}}\right\}$ where $J_{\mathbf{0}}(a)=\mathbf{0}, J_{\mathbf{1}}(a)=a$ for every $a \in E$. 
If $\mathcal{J}_{1}$ and $\mathcal{J}_{2}$ are compression bases for $E$, then $\mathcal{J}_{1} \cap \mathcal{J}_{2}$ is also a compression base for $E$. If $\left\{\mathcal{J}_{\alpha}\right\}$ is a chain of compression bases for $E$ then $\bigcup_{\alpha} \mathcal{J}_{\alpha}$ is also a compression base for $E$. As a consequence, according to Zorn's lemma, every effect algebra has a maximal compression base. If $J_{p}$ and $J_{p^{\prime}}$ are compressions, they are contained in a maximal compression base.

Let us present a prototypical example of an effect algebra with a compression base. Consider $H$ a Hilbert space and let $\mathcal{E}(H)$ be the set of all operators on $H$ that are self-adjoint, positive and smaller than identity. It is well known (see, e.g., [2]) that $\mathcal{E}(H)$ can be organised as an effect algebra with the partial operation defined by $A \oplus B=A+B$ if $A+B \in \mathcal{E}(H)$, for all $A, B \in \mathcal{E}(H)$. The set of sharp elements of this effect algebra is $\mathcal{P}(H)$, the set of projection operators on $H$. For every $P \in \mathcal{P}(H)$, let us define $J_{P}: \mathcal{E}(H) \rightarrow \mathcal{E}(H)$ by $J_{P}(A)=P A P$ for every $A \in \mathcal{E}(H)$. Then $\left(J_{P}\right)_{P \in \mathcal{P}(H)}$ is a compression base for $\mathcal{E}(H)$. Clearly, the focus of each compression $J_{P}$ is $P$, therefore the set of projections (in the sense of foci of compressions) of $\mathcal{E}(H)$ is just $\mathcal{P}(H)$.

Let us now summarize the properties of compressions that we intend to use in the sequel. They are direct consequences of the definition and of [5, Lemmas 3.1-3.3].

Lemma 3.4 Let $E$ be an effect algebra, $J$ a compression on $E$ with the focus $p$ and let us denote $p \circ a=J(a)$ for every $a \in E$. Then, for every $a, b \in E$ : (1) $p, p^{\prime}$ are principal and hence sharp; (2) $p \circ(a \oplus b)=(p \circ a) \oplus(p \circ b) ;(3) p \circ a \leq p \circ b$ whenever $a \leq b$; (4) $p \circ \mathbf{0}=\mathbf{0}, p \circ \mathbf{1}=p$; (5) $p \circ a=a$ if $a \leq p$; (6) $p \circ a \leq p$; $p \circ a=p$ if and only if $p \leq a$; (7) $p \circ a=\mathbf{0}$ if and only if $p \perp a\left(a \leq p^{\prime}\right)$.

For an effect algebra $E$ with a compression base $\left(J_{p}\right)_{p \in P}$ we denote:

- $p \circ a=J_{p}(a)$ for every $p \in P$ and $a \in E$;

- $p \mid q$ if $p, q \in P$ and $p \circ q=q \circ p$ (i.e., $\left.J_{p}(q)=J_{q}(p)\right)$;

- $C(p)=\left\{a \in E: a=J_{p}(a) \oplus J_{p^{\prime}}(a)\right\}$ for every $p \in P$.

Lemma 3.5 [6, Lemma 3.5] Let $\left(J_{p}\right)_{p \in P}$ be a compression base for the effect algebra $E$. Then $P$ is an orthomodular poset and $J_{p^{\prime}}$ is a supplement of $J_{p}$ for every $p \in P$.

Theorem 3.6 [6, Theorem 3.6] Let $E$ be an effect algebra with a compression base $\left(J_{p}\right)_{p \in P}$. For every $p, q \in P$, the following statements are equivalent: (1) $p \leq q$; (2) $J_{q} \circ J_{p}=J_{p}$; (3) $q \circ p=p$; (4) $J_{p} \circ J_{q}=J_{p}$; (5) $p \circ q=p$.

Theorem 3.7 [6, see Theorem 4.2] Let $E$ be an effect algebra with a compression base $\left(J_{p}\right)_{p \in P}$. For every $p, q \in P$, the following statements are equivalent: (1) $p \circ q=q \circ p$; (2) $p$ and $q$ coexist; (3) $p \in C(q)$.

Let us now briefly present sequential effect algebras which will be regarded here as a particular case of effect algebras with a compression base. A detailed account regarding sequential effect algebras can be found in [7].

Definition 3.8 A sequential product on an effect algebra $(E, \oplus, \mathbf{0}, \mathbf{1})$ is a binary operation ○ on $E$ such that for every $a, b, c \in E$, the following conditions hold:

(S1) $a \circ(b \oplus c)=(a \circ b) \oplus(a \circ c)$ if $b \oplus c$ exists;

(S2) $1 \circ a=a$;

(S3) if $a \circ b=\mathbf{0}$ then $a \mid b$ (where $a \mid b$ denotes $a \circ b=b \circ a$ );

(S4) if $a \mid b$ then $a \mid b^{\prime}$ and $a \circ(b \circ c)=(a \circ b) \circ c$;

(S5) if $c \mid a, b$ then $c \mid a \circ b$ and $c \mid(a \oplus b)$ (if $a \oplus b$ exists).

An effect algebra endowed with a sequential product is called a sequential effect algebra. 
The definition of sequential effect algebras was inspired by the so-called standard Hilbert space effect algebra which is exactly $\mathcal{E}(H)$, previously described. More precisely, on $\mathcal{E}(H)$ a sequential product is defined by $A \circ B=A^{1 / 2} B A^{1 / 2}$ for $A, B \in \mathcal{E}(H)$.

Theorem 3.9 [6, Theorem 3.4] Let $E$ be a sequential effect algebra. For every $p \in E_{\mathrm{s}}$, the mapping $J_{p}: E \rightarrow E$ defined by $J_{p}(a)=p \circ a$ is a compression with the focus $p$. The system $\left(J_{p}\right)_{p \in E_{\mathrm{s}}}$ is a maximal compression base for $E$.

In view of the above theorem, it should be clear that the notation $p \circ a=J_{p}(a)$ (as well as $p \mid q$ for $p \circ q=q \circ p$ ) introduced for general effect algebras with a compression base is inspired by the particular case of sequential effect algebras. However, in the general case of an effect algebra $E$ with a compression base $\left(J_{p}\right)_{p \in P}$, the (partial) operation $\circ: P \times E \rightarrow E$ defined by $p \circ a=J_{p}(a)$ need not be the restriction of a sequential product on $E$ (see [5]).

\section{Atoms and centrality}

Proposition 4.1 Let $E$ be an effect algebra. If $p$ is an atom in $E$ that is the focus of a compression and $a \in E$ then $p \leq a$ or $p \leq a^{\prime}$.

Proof: Since $p \circ a \leq p$ and $p$ is an atom, either $p \circ a=\mathbf{0}$ or $p \circ a=p$. If $p \circ a=\mathbf{0}$ then, according to Lemma 3.4, $p \perp a$, hence $p \leq a^{\prime}$. If $p \circ a=p$, then $p \circ a=p=p \circ \mathbf{1}=p \circ\left(a \oplus a^{\prime}\right)=$ $(p \circ a) \oplus\left(p \circ a^{\prime}\right)$. Applying the cancellation law, $p \circ a^{\prime}=\mathbf{0}$, hence, according to Lemma 3.4 $p \perp a^{\prime}$ and therefore $p \leq a$.

Corollary 4.2 Distinct atoms that are foci of compressions in an effect algebra are orthogonal.

Corollary 4.3 Let $E$ be an effect algebra with a compression base $\left(J_{p}\right)_{p \in P}$. If $p, q \in P$ and $p$ is an atom in $E$ then $p \mid q$.

Proof: According to Proposition 4.1, $p \leq q$ or $p \leq q^{\prime}$. In the first case, according to Theorem 3.6, $p \circ q=p=q \circ p$, hence $p \mid q$. If $p \leq q^{\prime}$, then $p \perp q$ and, according to Lemma 3.4. $p \circ q=\mathbf{0}=q \circ p$, hence $p \mid q$.

Proposition 4.4 Let $E$ be an effect algebra with a compression base $\left(J_{p}\right)_{p \in P}$ and $p, q, r \in P$ such that $p \leq q \circ r$ and $p \mid r$. Then $p \leq r \circ q$.

Proof: According to Lemma 3.4, $p \leq q$. According to Lemma 3.4, Theorem 3.6, the assumption and Lemma 3.4 again, $p=p \circ(q \circ r)=J_{p}\left(J_{q}(r)\right)=J_{p}(r)=p \circ r=r \circ p \leq r \circ q$.

Proposition 4.5 Let $E$ be an effect algebra with a compression base $\left(J_{p}\right)_{p \in P}$ and $p \in P$ be an atom in $E$. For every $q, r \in P, p \leq q \circ r$ if and only if $p \leq r \circ q$.

Proof: This is a straightforward consequence of Corollary 4.3 and Proposition 4.4

Theorem 4.6 Let $E$ be an effect algebra with a compression base $\left(J_{p}\right)_{p \in P}$. If $E$ is determined by atoms and every atom is in $P$ then $P$ is a Boolean algebra.

Proof: Let $q, r \in P$. According to Proposition 4.5, $q \circ r$ and $r \circ q$ dominate the same set of atoms (since all atoms are in $P$ ). Since $E$ is determined by atoms, this means $q \circ r=r \circ q$ and hence, according to Theorem 3.7, $q, r$ coexist. According to Lemma 3.5, $P$ is an OMP. Hence, $P$ is an OMP with every pair of its elements coexistent and therefore a Boolean algebra (see, e.g., [8, Theorem 1.3.13]). 
Let us remark that the conclusion of the above theorem cannot be improved to the statement that $E$ is a Boolean algebra. The effect algebra in Example 5.12 satisfies the hypotheses (it is even atomistic), however it is not a Boolean algebra.

Lemma 4.7 Let $E$ be an effect algebra with a compression base $\left(J_{p}\right)_{p \in P}$. If $p \in P$ is an atom in $E$ then $C(p)=E$.

Proof: Let $a \in E$. According to Proposition 4.1, $p \leq a$ or $p \leq a^{\prime}$.

If $p \leq a$ (and therefore $\left.a^{\prime} \leq p^{\prime}\right)$, then $J_{p}(a)=p$ and $J_{p^{\prime}}\left(a^{\prime}\right)=a^{\prime}$ and therefore $J_{p}(a) \oplus$ $J_{p^{\prime}}(a)=p \oplus J_{p^{\prime}}\left(\mathbf{1} \ominus a^{\prime}\right)=p \oplus\left(p^{\prime} \ominus a^{\prime}\right)=p \oplus(a \ominus p)=a$.

If $p \leq a^{\prime}$ (and thus $a \leq p^{\prime}$ ), then $J_{p^{\prime}}(a)=a, J_{p}(a)=\mathbf{0}$ and thus $J_{p}(a) \oplus J_{p^{\prime}}(a)=a$.

Remark 4.8 Let $E$ be an effect algebra with a compression base $\left(J_{p}\right)_{p \in P}$. The previous result implies that every atom $p \in P$ in $E$ coexists with every element of $E$. Indeed, for every $a \in E=C(p), a=J_{p}(a) \oplus J_{p^{\prime}}(a)$. Since $J_{p}(a) \leq p$, there is a $p_{1} \in E$ such that $p=J_{p}(a) \oplus p_{1}$. Taking into account that $J_{p^{\prime}}(a) \leq p^{\prime}$, it follows that the sum $J_{p^{\prime}}(a) \oplus p=J_{p^{\prime}}(a) \oplus J_{p}(a) \oplus p_{1}$ exists and therefore $a$ and $p$ coexist.

The following result that will be useful in the sequel can be deduced from [6, Lemma 4.1]. However, we will present a different proof for it.

Lemma 4.9 Let $E$ be an effect algebra with a compression base $\left(J_{p}\right)_{p \in P}$. An element $p \in P$ is a central element of $E$ if and only if $C(p)=E$.

Proof: If $C(p)=E$, then $a=J_{p}(a) \oplus J_{p^{\prime}}(a)$, for every $a \in E$. According to Lemma 3.4 $p, p^{\prime}$ are principal, $J_{p}(a) \leq p$ and $J_{p^{\prime}}(a) \leq p^{\prime}$, hence $p$ is a central element of $E$.

Conversely, let us suppose that $p$ is a central element of $E$. For every $a \in E$ there are $a_{1} \leq p$, $a_{2} \leq p^{\prime}$ such that $a=a_{1} \oplus a_{2}$. Hence $J_{p}(a)=J_{p}\left(a_{1} \oplus a_{2}\right)=J_{p}\left(a_{1}\right) \oplus J_{p}\left(a_{2}\right)=a_{1} \oplus \mathbf{0}=a_{1}$ and similarly $J_{p^{\prime}}(a)=a_{2}$. Thus $a=a_{1} \oplus a_{2}=J_{p}(a) \oplus J_{p^{\prime}}(a)$ and it follows that $a \in C(p)$.

Remark 4.10 In particular, the above lemma holds if $E$ is a sequential effect algebra endowed with the compression base $\left(J_{p}\right)_{p \in E_{\mathrm{s}}}, J_{p}(a)=p \circ a$.

Theorem 4.11 Let $E$ be an effect algebra with a compression base $\left(J_{p}\right)_{p \in P}$. Every $p \in P$ that is an atom in $E$ is central in $E$.

Proof: Let $p \in P$ be an atom in $E$. According to Lemma 4.7, $C(p)=E$ and, according to Lemma 4.9, $p$ is central in $E$.

\section{Projection-atomic effect algebras}

The following property is intended as a substitute, in the framework of atomic effect algebras having a compression base, for the property, in an effect algebra, of having all the atoms sharp.

Definition 5.1 An effect algebra $E$ is projection-atomic if it is atomic and there is a compression base $\left(J_{p}\right)_{p \in P}$ of $E$ such that $P$ contains all atoms in $E$.

In view of the above definition, the result of Theorem 4.11 implies that atoms of a projection-atomic effect algebra are central. The converse also holds, as will be shown in the next remark. 
Remark 5.2 Pulmannová [9, Example 3.4] proved that for every effect algebra $E$ the center $\widetilde{C}(E)$ is a normal sub-EA and $\left(J_{p}\right)_{p \in \widetilde{C}(E)}$ with $J_{p}(a)=p \wedge a$ is a compression base. Hence, every atomic effect algebra with all atoms central is projection-atomic.

\section{Proposition 5.3 Every projection-atomic effect algebra is an orthoalgebra.}

Proof: Let $E$ be a projection-atomic effect algebra. Then $E$ is atomic and, according to Theorem 4.11, all its atoms are central, hence sharp. According to Proposition 2.9, $E$ is an orthoalgebra.

The following properties of an effect algebra $E$ will be useful in the sequel:

Definition 5.4 A subset $M$ of an effect algebra $E$ is downward directed if for every $a, b \in M$ there is an element $c \in M$ such that $c \leq a, b$.

An effect algebra $E$ has the maximality property if $[\mathbf{0}, a] \cap[\mathbf{0}, b]$ has a maximal element for every $a, b \in E$.

An effect algebra $E$ is weakly distributive if $a \wedge b=a \wedge b^{\prime}=\mathbf{0}$ implies $a=\mathbf{0}$ for every $a, b \in E$.

Remark 5.5 The maximality property generalizes several important properties of effect algebras, e.g., every chain-finite, orthocomplete or lattice effect algebra has the maximality property. For details and more properties generalized by the maximality property see [12, Theorem 4.1] and [13, Theorem 3.1].

Theorem 5.6 [10, Theorem 4.2] Every weakly distributive orthomodular poset with the maximality property is a Boolean algebra.

\section{Lemma 5.7 Every projection-atomic effect algebra is weakly distributive.}

Proof: Let $E$ be a projection-atomic effect algebra and $\left(J_{p}\right)_{p \in P}$ a compression base of $E$ such that $\mathrm{P}$ contains all atoms in $E$. Suppose that $E$ is not weakly distributive. Then there are $a, b \in E$ such that $a \neq \mathbf{0}, a \wedge b=\mathbf{0}$ and $a \wedge b^{\prime}=\mathbf{0}$. Since $E$ is projection-atomic, there is an atom $p \in P$ in $E$ such that $p \leq a$. Then $p \not \leq b$ and $p \not \leq b^{\prime}$, which contradicts to Proposition 4.1

Lemma 5.8 The set of upper bounds of a set of atoms in a projection-atomic effect algebra with the maximality property is downward directed.

Proof: Let $E$ be a projection-atomic effect algebra with a compression base $\left(J_{p}\right)_{p \in P}$ such that $\mathrm{P}$ contains the set of atoms of $E, A \subset P$ be a set of atoms, $a, b$ be upper bounds of $A$. According to the maximality property, there is a maximal $c \leq a, b$. Let us suppose that $c$ is not an upper bound of $A$ and seek a contradiction. Then there is an atom $d \in A$ such that $d \not \leq c$, hence, according to Proposition 4.1. $d \leq c^{\prime}$ and therefore $d^{\prime} \geq c$. Since $d \leq a, b$ and therefore $d^{\prime} \geq a^{\prime}, b^{\prime}, c \perp a^{\prime}, b^{\prime}$ and $d^{\prime}$ is central (Theorem 4.11) and therefore principal, we obtain $d^{\prime} \geq c \oplus a^{\prime}$ and $d^{\prime} \geq c \oplus b^{\prime}$. Hence $d \leq\left(c \oplus a^{\prime}\right)^{\prime}=a \ominus c$ and $d \leq\left(c \oplus b^{\prime}\right)^{\prime}=b \ominus c$ and therefore $c \oplus d \leq a, b$-which contradicts the maximality of $c$.

Lemma 5.9 Every element in a projection-atomic effect algebra is a minimal upper bound of the set of atoms it dominates. Every projection-atomic effect algebra with the maximality property is atomistic.

Proof: Let $E$ be a projection-atomic effect algebra, $a \in E$ and $A_{a}$ be the set of atoms dominated by $a$. First, let us show that $a$ is a minimal upper bound of $A_{a}$. Let us suppose that 
there is an upper bound $b<a$ of $A_{a}$ and seek a contradiction. Then $a \ominus b \neq \mathbf{0}$ and since $E$ is atomic, there is an atom $p \in A_{a}$ such that $p \leq a \ominus b$ and therefore $p \leq b^{\prime}$. Since $p \leq b$ and $E$ is an orthoalgebra (Proposition 5.3), we obtain $p \leq b \wedge b^{\prime}=\mathbf{0}$ - a contradiction.

If $E$ has the maximality property then, according to Lemma 5.8, the set of upper bounds of $A_{a}$ is downward directed, hence $a=\bigvee A_{a}$.

Lemma 5.10 Every projection-atomic effect algebra with the maximality property is an orthomodular poset.

Proof: Let $E$ be a projection-atomic effect algebra with the maximality property, $a, b \in E$ with $a \perp b$ and $A_{a}, A_{b}$ be the sets of atoms dominated by $a$ and $b$ respectively. According to Lemma 5.9, $E$ is atomistic and therefore the set of upper bounds of $\{a, b\}$ is the set of upper bounds of $A_{a} \cup A_{b}$. According to Proposition 5.3, $E$ is an orthoalgebra and therefore $a \oplus b$ is a minimal upper bound of $\{a, b\}$ ([2, Theorem 5.1]). According to Lemma 5.8, the set of upper bounds of $A_{a} \cup A_{b}$ is downward directed, hence $a \oplus b$ is the least upper bound of $\{a, b\}$. Hence $a \oplus b=a \vee b$ for orthogonal $a, b \in E$ and therefore $E$ is an orthomodular poset (see [3, Theorem 2.12]).

Theorem 5.11 Every projection-atomic effect algebra with the maximality property is a Boolean algebra.

Proof: It follows from Lemma 5.7, Lemma 5.10 and Theorem 5.6.

We can replace the maximality property in Theorem 5.11 by various stronger properties (see Remark 5.5), e.g., by the orthocompleteness. It cannot be replaced by the weak orthocompleteness, as the following example based on Tkadlec [11, 13] shows.

Example 5.12 Let $X_{1}, X_{2}, X_{3}, X_{4}$ be infinite and mutually disjoint sets, $X=\bigcup_{i=1}^{4} X_{i}$,

$$
\begin{aligned}
E^{\prime} & =\left\{\emptyset, X_{1} \cup X_{2}, X_{2} \cup X_{3}, X_{3} \cup X_{4}, X_{4} \cup X_{1}, X\right\}, \\
E & =\left\{(A \backslash F) \cup(F \backslash A): A \in E^{\prime} \text { and } F \subset X \text { is finite }\right\} .
\end{aligned}
$$

For disjoint $A, B \in E$ we define $A \oplus B=A \cup B$. Then $(E, \oplus, \emptyset, X)$ is an orthomodular poset, the orthosupplement is the set theoretic complement in $X$ and the partial ordering is the inclusion. $E$ is atomic and the set of its atoms is $\{\{x\}: x \in X\}$. Let us put

$$
P=\{F \subseteq X: F \text { is finite or } X \backslash F \text { is finite }\}
$$

and for every $F \in P$ let us define $J_{F}: E \rightarrow E$ by $J_{F}(A)=F \cap A$ for every $A \in E$.

It is a straightforward verification that $\left(J_{F}\right)_{F \in P}$ is a compression base for $E$ and that $P$ contains all atoms, hence $E$ is projection-atomic. $E$ is weakly orthocomplete, because if an orthogonal system $\left(A_{i}\right)_{i \in I}$ has a minimal majorant $B \in E$ then $B=\bigcup_{i \in I} A_{i}$ is the sum of $\left(A_{i}\right)_{i \in I}$. Since all elements of $\left[\emptyset, X_{2}\right]$ are finite, $\left(X_{1} \cup X_{2}\right) \wedge\left(X_{2} \cup X_{3}\right)$ does not exist and therefore $E$ is not a lattice (and hence not a Boolean algebra).

Let us remark that $P \neq E-$ e.g., $X_{1} \cup X_{2} \in E \backslash P$.

Definition 5.13 A compression base $\left(J_{p}\right)_{p \in P}$ on an effect algebra $E$ has the projection cover property [6] if for every element $a \in E$ there exists the least element $b \in P$ (the projection cover of $a$ ) with $b \geq a$.

Theorem 5.14 Let $E$ be a projection-atomic effect algebra. If a compression base on $E$ for which all atoms are projections has the projection cover property, then $E$ is a Boolean algebra. 
Proof: Let $\left(J_{p}\right)_{p \in P}$ be a compression base on $E$ that has the projection cover property and such that all atoms are in $P$. According to [9, Theorem 5.1], $P$ is an orthomodular lattice. Since $P$ is atomic, it is atomistic (see, e.g., [8]). Since all atoms are mutually orthogonal (see Corollary 4.2), every two elements of $P$ are compatible, and hence $P$ is a Boolean algebra.

It remains to prove that $E=P$. Let $a \in E$ and let us denote $A_{a}$ the set of atoms in $E$ dominated by $a$ and $P_{a}=\{p \in P: p \leq a\}$. The set of projection upper bounds of $a^{\prime}$ is $P_{a}^{\prime}=\left\{p^{\prime} \in P: p \in P_{a}\right\}$ and, due to the projection cover property, there is a projection cover $\bigwedge P_{a}^{\prime} \in P$ of $a^{\prime}$, hence $a \geq \bigvee P_{a} \in P$. Since $a$ is a minimal upper bound of $A_{a}$ (Lemma 5.9) and $\bigvee P_{a}$ is also an upper bound of $A_{a}$, it follows that $a=\bigvee P_{a} \in P$.

\section{Corollary 5.15 Every atomic sequential orthoalgebra is a Boolean algebra.}

Proof: According to Theorem 3.9, every sequential effect algebra $E$ has a maximal compression base $\left(J_{p}\right)_{p \in E_{\mathrm{s}}}$. If $E$ is an orthoalgebra then $E=E_{\mathrm{s}}$ and therefore every element of $E$ is its own projection cover, hence, according to Theorem 5.14, $E$ is a Boolean algebra.

Let us remark that the above corollary generalizes similar results obtained by Gudder and Greechie [7, Theorem 5.3] and Tkadlec [11, Theorems 5.4 and 5.6]. The first mentioned result assumes that the effect algebra is atomistic, the second assumes it has the maximality property and the third assumes it is determined by atoms.

\section{Acknowledgments}

The first author was supported by the scholarship of Czech Technical University and the second author by the research plan of the Ministry of Education of the Czech Republic No. 6840770010 .

\section{References}

1. Dvurečenskij, A., Pulmannová, S.: New Trends in Quantum Structures. Kluwer Academic, Bratislava (2000).

2. Foulis, D. J., Bennett, M. K.: Effect algebras and unsharp quantum logics. Found. Phys. 24, 13311352 (1994). doi:10.1007/BF02283036

3. Foulis, D. J., Greechie, R. J., Rüttimann, G. T.: Filters and supports in orthoalgebras. Int. J. Theor. Phys. 31, 789-807 (1992). doi:10.1007/BF00678545

4. Greechie, R. J.: A particular non-atomistic orthomodular poset. Commun. Math. Phys. 14, 326-328 (1969). doi:10.1007/BF01645388

5. Gudder, S.: Compressible effect algebras Rep. Math. Phys. 54, 93-114 (2004). doi:10.1016/S00344877(04)80008-9

6. Gudder, S.: Compression bases in effect algebras. Demonstr. Math. 39, 43-54 (2006).

7. Gudder, S., Greechie, R. J.: Sequential products on effect algebras. Rep. Math. Phys. 49, 87-111 (2002). doi:10.1016/S0034-4877(02)80007-6

8. Pták, P., Pulmannová, S.: Orthomodular Structures as Quantum Logics. Kluwer Academic Publ. Dordrecht and VEDA, Bratislava (1991).

9. Pulmannová, S.: Effect algebras with compressions. Rep. Math. Phys. 58, 301-324 (2006). doi:10.1016/S00344877(06)80054-6

10. Tkadlec, J.: Conditions that force an orthomodular poset to be a Boolean algebra. Tatra Mt. Math. Publ. 10, 55-62 (1997).

11. Tkadlec, J.: Atomic sequential effect algebras. Int. J. Theor. Phys. 47, 185-192 (2008). doi:10.1007/s10773007-9492-1

12. Tkadlec, J.: Effect algebras with the maximality property. Algebra Universalis 61, 187-194 (2009). doi:10.1007/s00012-009-0013-3

13. Tkadlec, J.: Common generalizations of orthocomplete and lattice effect algebras. Int. J. Theor. Phys. 49 (2010), 3279-3285. doi:10.1007/s10773-009-0108-9 